\title{
Measurements of the Planck Length from a Ball Clock without Knowledge of Newton's Gravitational Constant $G$ or the Planck Constant
}

\author{
Espen Gaarder Haug
}

\begin{abstract}
We demonstrate how one can extract the Planck length from a ball with a built-in stopwatch, without knowledge of the Newtonian gravitational constant or the Planck constant. This could be of great importance since, until recently, it has been assumed the Planck length cannot be found without knowledge of Newton's gravitational constant. This method of measuring the Planck length should also be of great interest not only to physics researchers but also to physics teachers and students, as it conveniently demonstrates that the Plank length is directly linked to gravitational phenomena, not only theoretically but practically. To demonstrate that this is more than a theory, we report 100 measurements of the Planck length using this simple approach. We will claim that, despite the mathematical and experimental simplicity, our findings could be of great importance in better understanding the Planck scale and quantum gravity, as our findings strongly support the idea that to detect gravity is to detect the effects from the Planck scale indirectly.
\end{abstract}

Index Terms-Planck length, Planck units, Newton's gravitational constant, Planck constant, Compton wavelength.

\section{INTRODUCTION}

In 1899 and 1906, Max Planck [1], [2] assumed there were three important universal constants: the speed of light, the Planck constant, and the Newtonian gravitational constant. Based on these, he used dimensional analysis and predicted there had to be a very fundamental length, $l_{p}=\sqrt{\frac{G \hbar}{c^{3}}}$; time unit, $t_{p}=\sqrt{\frac{G \hbar}{c^{5}}}$, and mass, $m_{p}=\sqrt{\frac{\hbar c}{G}}$. Still, what were these Planck units related to? Max Planck said little about this, except for calling them natural units. He had also not been the first to suggest natural units. Stoney [3] had, in 1883, already suggested natural units derived from the elementary charge, the Newtonian gravitational constant, and the speed of light. Today, however, most physicists consider the Planck units to be more important than the Stoney units.

The Planck units were in no way immediately accepted as something important, and it was mainly the Planck constant (quanta of energy) and Planck's law that made Max Planck very famous in physics and won him a Nobel Prize. The Planck units are today considered to potentially play an important role in a "final" unified theory. Already, by 1916, Einstein [4] had laid out his general relativity theory in one of his papers, indicating that a quantum gravity theory was the next natural

DOI: http://dx.doi.org/10.24018/ejphysics.2021.3.6.133

Published on December 02, 2021.

E. G. Haug is with the Norwegian University of Life Sciences (ORCID: 0000-0001-5712-609) step to an even better understanding of gravity or, in his own words:

Because of the intra-atomic movement of electrons, the atom must radiate not only electromagnetic but also gravitational energy, if only in minute amounts. Since, in reality, this cannot be the case in nature, then it appears that the quantum theory must modify not only Maxwell's electrodynamics but also the new theory of gravitation. (Einstein 1916, p. 696).

However, Einstein said little or nothing about how the quantum theory should cause modifications in gravity theory. Eddington [5], in 1918, is likely to have been the first to suggest that the Planck length must play a central role in new quantum gravity theory or, in his own words:

There are three fundamental constants of nature which stand out as pre-eminent. The velocity of light, $300 \cdot 10^{10}$ C.G.S. units ; dimensions $L T^{-1}$. The quantum, $6.55 \cdot 10^{-27}, M L^{2} T^{-1}$ C.G.S. Units. The constant of gravitation, $6.66 \cdot 10^{-8} ; M^{-1} L^{2} T^{-2}$. From these we can construct a fundamental unit of length whose value is $4 \times 10^{-33} \mathrm{~cm}$. There are other natural units of length, the radii of the positive and negative unit electric charges, but these are of an altogether higher order of magnitude. But it is evident that this length must be the key to some essential structure. It may not be an unattainable hope that someday a clearer knowledge of the process of gravitation may be reached.

It is clear that it is the Planck length that Eddington refers to. However, this "speculation" that gravity likely had to be linked to the Planck length was by no means easily accepted. For example, Bridgman [6] ridiculed Eddington in 1931 for his speculative claim, and he himself basically claimed the Planck units were more of a mathematical artifact coming out of dimensional analysis rather than something linked to anything physical. Today most physicists, especially those working with gravity and quantum gravity theories, [7]-[12] seem to agree with Eddington that the Planck length must likely play central role in a quantum gravity - in addition, superstring theorists assume the Planck length is important for their theory. Still, there are others [13] that are more inclined to agree with Bridgman and think of the Planck units more like a mathematical artifact, or at least something we can never measure except by deriving it through dimensional analysis. However, 
recently, we [15], [16] have shown how one can measure the Planck length and Plank time independent of $G$ and $\hbar$ by using a Newton force spring, and also how to find the Planck length independent of $G$ by using a Cavendish apparatus [14], although these methods are somewhat more complicated than shown here. Here, we will extend our analysis to measure the Planck length with a ball with a built-in stopwatch-this makes it particularly easy to perform such an experiment, so that even high school physics students can obtain a better understanding of how one can extract the Planck length from gravity phenomena.

\section{The Compton Wavelength And Counting Atoms}

Our method will rely on the fact that we know the Compton wavelength of the gravity object from which we will measure the gravity effects. First, we start out by measuring the Compton wavelength based on Compton scattering, as described by Compton [17] in 1923. Compton scattering consists of shooting photons at an electron and measuring the wavelength of the photon before $\lambda_{\gamma, 1}$ and after $\lambda_{\gamma, 1}$ it hits the electron, as well as measuring the angle between the ingoing and outgoing photon $(\theta)$.

$$
\lambda_{e}=\frac{\lambda_{\gamma, 2}-\lambda_{\gamma, 1}}{1-\cos \theta}
$$

It is important to note that this way of measuring the Compton wavelength of the electron requires no knowledge of the Planck constant. We could alternatively have found the Compton wavelength from the electron mass in kilograms $(\mathrm{kg})$, but this would generally require knowledge of the Planck constant. This is given by the well-known Compton wavelength formula: $\lambda=\frac{h}{m c}$; however, we will stick to the first method that is independent of knowledge of the Planck constant. Next, we will find the Compton wavelength of a proton. We then utilize the fact that the Compton wavelength is inversely linearly proportional to the cyclotron frequency. This because the cyclotron frequency is given by:

$$
f=\frac{q B}{2 \pi m}
$$

and since the charge of a proton and electron is the same, the relative cyclotron frequency of a proton relative to an electron is equal to the Compton wavelength ratio; that is to say, we have:

$$
\frac{f_{e}}{f_{P}}=\frac{\frac{q B}{2 \pi m_{e}}}{\frac{q B}{2 \pi m_{P}}}=\frac{m_{P}}{m_{e}}=\frac{\lambda_{e}}{\lambda_{P}} \approx 1836.15
$$

This method has been used to accurately find the proton electron mass ratio [18], [19], which is identical to the Compton wavelength ratio. If we have now measured the electron Compton wavelength by Compton scattering, we simply need to divide this by the cyclotron frequency ratio to find the Compton wavelength of the proton. Theories about the Compton wavelength of the proton reach back to, at least, Levitt [20] in 1958, who claimed:

Most of the experimental lengths concerning the fundamental forces in the nucleus are integral multiples of the Compton wavelength, $\lambda_{0}$, of the proton, where $\lambda_{0}=\frac{h}{m_{0} c}=1.32 \times 10^{-13} \mathrm{~cm}$ (which we can thus call a 'Compton'). These lengths include the effective range of nucleon interactions, and the amplitudes of dispersion.

The author uses notation $\lambda_{0}$ for the Compton wavelength of the rest mass, but we have simply used notation $\lambda$. We also note an increased interest in the Compton wavelength of the proton, which has recently, for example, been suggested to also be directly related to the proton radius [21]. Some will possibly protest here and claim only elementary particles like the electron have a Compton wavelength, and that protons do not. We, to a large degree, agree with such an argument, but even if a composite particle or even a large composite mass does not have one single Compton wavelength, they ultimately consist of many elementary particles each with a Compton wavelength. The aggregates of the individual Compton wavelengths in any mass are given by the relation below (see also [15])

$$
\lambda=\frac{1}{\sum_{i}^{n} \frac{1}{\lambda_{i}}}
$$

Since we already know how to find the Compton wavelength of a proton (without knowledge of $h$ ) all we now need to do to find the Compton wavelength of, for example, a handful of matter is to count the number of atoms in that clump of matter. This method has been used since the early history of physics, at least to Avogadro's number [22]. It is not easy, but we can indeed count the number of atoms very accurately today. For example, one of the new $\mathrm{kg}$ definition suggestions was rooted in counting atoms in silicon spheres [23], [24], [27]. But then what about really large masses such as the Earth? How can we count the number of atoms in the Earth? Here we can take advantage of the fact that the ratio of the Compton wavelength in two masses is always linearly proportional to almost any two gravitational measurements performed on the two objects, after correcting for distances to the centre of the gravity objects. For example, if we know the gravitational acceleration from a small silicon sphere and the gravitational acceleration field of the Earth, then we know that the Compton wavelength in the Earth is related to:

$$
\frac{g_{1} R_{1}^{2}}{g_{2} R_{2}^{2}}=\frac{\lambda_{2}}{\lambda_{1}}
$$

To predict the gravitational acceleration from the mass of the Earth, in standard physics we need to know $G$ since we have $g=\frac{G M}{R^{2}}$, but we can measure the gravitational acceleration of the Earth, for example, at the surface of the Earth, without any knowledge of $G$. This we can do by dropping a ball from height $H$ to the ground and measuring how long this took, and based on this we know $g$ from the following well-known formula:

$$
g=\frac{2 H}{T^{2}}
$$

From a small mass (such as a silicon sphere) in which we been able to count the number of atoms, we can measure the gravitational acceleration field with a Cavendish apparatus based on the following relation: 


$$
g=\frac{2 \pi^{2} L \theta}{T^{2}}
$$

where $\theta$ is the angle of the arm when it is deflected, and $L$ is the distance between the two small balls in the apparatus, and $T$ is the oscillation time.

Pay attention to the fact that no $G$ or $\hbar$ are needed to find the gravitational acceleration field in the Cavendish apparatus. Newton [25] did not use or introduce a gravity constant. It is often said that the Cavendish apparatus was developed to measure $G$; however this is not the case. A Cavendish apparatus can be used to measure $G$, but in 1798 Cavendish [26] did not use or measure the gravitational constant $G$. What Cavendish did was to use the apparatus to find the gravitational effects from a uniform mass-in his case, lead balls-that he already knew the density of. When he knew this, he could find the density of the Earth relative to this. The gravity constant was actually first introduced in 1873 by Cornu and Baill [28] and was partly needed because the research community now had redefined the mass standard in terms of $\mathrm{kg}$; they actually used notation $f$ for the gravity constant, while in 1994 Boys [29] introduced today's notation $G$. Whether one uses $f$ or $G$ notation is naturally only a purely cosmetic question. The main point is that we can measure gravitational acceleration with no knowledge of $G$ and, if we have counted the number of atoms (protons and neutrons) in the reference mass, for example a silicon sphere, then we can find the Compton wavelength of even a planet or a sun without any knowledge of $G$ or $\hbar$. The Compton wavelength of the Earth is approximately $\lambda_{E} \approx 3.70 \times 10^{-67} \mathrm{~m}$, and the reduced Compton wavelength is this number divided by $2 \pi$, which gives approximately $\bar{\lambda}_{E} \approx 5.89 \times 10^{-68} \mathrm{~m}$. Again, this is not a physical single Compton wavelength, but corresponds to the aggregate of the Compton wavelength of all elementary particles making up the mass of the Earth, see formula 4.

\section{Planck Length measured With A BALL-CLOCK}

It is well known that one can measure $g$ by measuring the velocity of a ball from a drop height $H$, as shown in the section above. Traditionally, one needs two synchronized clocks to do this: one clock that measures when the ball is dropped from point $H$, and one clock measuring when the ball hits the ground or passes a point below $H$. That is to say, an external observer frame observes the time it takes for the object to fall, and therefore to be accelerated by the gravitational acceleration field. In recent years, this has become much easier to do as we have instead incorporated a stopwatch inside the ball itself as, in other words, a proper observer. What is known as a "gravity ball" or just "g-ball" is a ball with a built-in stopwatch that starts at the moment one drops the ball and stops when the ball hits something, such as the ground. Such a ball costs about 30 USD at the time of writing, and it has about one-hundredth of a second precision. If we know the Compton wavelength of the Earth, then we can use such a ball to measure the Planck length in a very simple way. This new device makes it easy to measure the gravitational acceleration field that again is given by:

$$
g=\frac{2 H}{T^{2}}
$$

as we also have $g=\frac{G M}{R^{2}}$ and in addition we can solve the Planck length formula, $l_{p}=\sqrt{\frac{G \hbar}{c^{3}}}$ that gives, with respect to $G$, this:

$$
G=\frac{l_{p}^{2} c^{3}}{\hbar}
$$

To make the gravity constant a function of the Planck length would naturally only lead to a circular unsolvable problem if we cannot find the Planck length independent of knowledge of $G$. The idea that the Newtonian gravitational constant is a composite constant expressed through Planck units is not new. Already in 1984, Cahill [33] suggested that one could express the Newton gravitational constant as $G=\frac{\hbar c}{m_{p}^{2}}$, which is simply the Planck mass formula solved with respect to $G$ but, as pointed out by Cohen [34] in 1987, this only led to a circular problem as one had to know $G$ to find $m_{p}$. This has, until recently, been the view among researchers, and this view was repeated as late as in 2016 in an interesting paper by McCulloch [35]. It is when we can first find the Planck units independent of $G$ that we really can claim $G$ must be a composite constant and that the Planck units play a more important role, something we have recently demonstrated in 2017 and 2020, and also will demonstrate in this paper in a very simple and powerful way using a ball with a built in stopwatch.

Next, we can solve the Compton 1923 wavelength formula, $\lambda=\frac{h}{m c}$ which, with respect to the mass, gives:

$$
m=\frac{h}{\lambda} \frac{1}{c}
$$

To solve the Compton wavelength formula with respect to $m$ is trivial, but we have not seen the kilogram mass expressed in this way before 2018 [36]. Further the gravitational acceleration is then given by:

$$
g=\frac{G m}{R^{2}}=\frac{l_{p}^{2} c^{3}}{\hbar} \frac{\frac{h}{\lambda} \frac{1}{c}}{R^{2}}=c^{2} \frac{l_{p}^{2}}{\bar{\lambda} R^{2}}
$$

As we can measure $g$ without knowing $G$ or $m$, we can now solve this equation with respect to $l_{p}$; this gives:

$$
l_{p}=\frac{R}{c} \sqrt{g \bar{\lambda}}
$$

Next we replace $g$ with $g=\frac{2 H}{T^{2}}$, and this gives:

$$
l_{p}=\frac{R}{c T} \sqrt{2 H \bar{\lambda}}
$$

That is to say, we can now measure the Planck length from dropping a ball with a built-in stopwatch, as $T$ is the drop time and $H$ is the height of the drop; furthermore, $R$ is the radius of the Earth and $c$ is the speed of light that we can look up or measure independently. Figure 1 shows a picture of our drop-ball with built-in stopwatch (also known as a g-ball).

We dropped this ball 100 times from a height of approximately two meters. After each ball drop, we read the time 


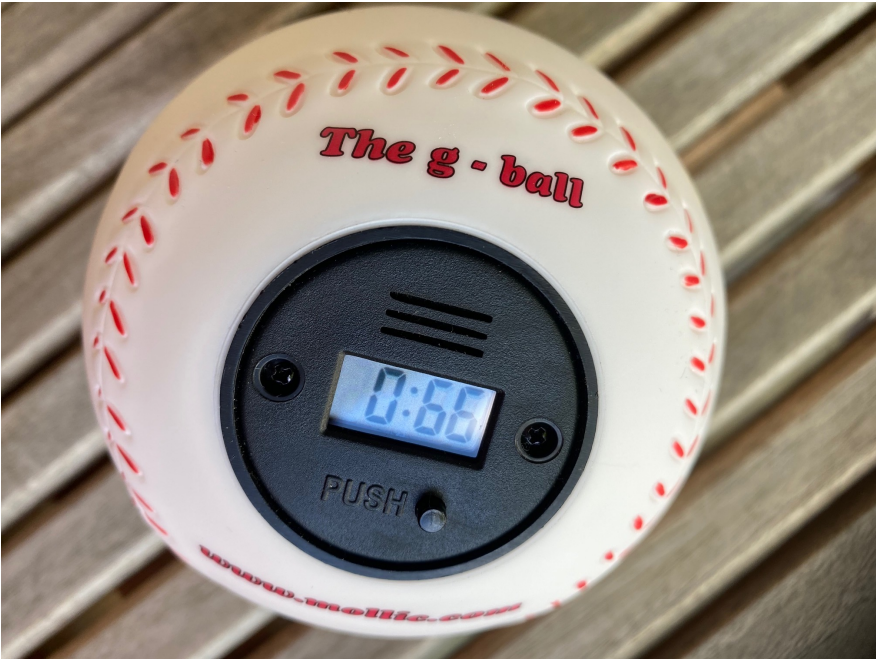

Fig. 1. The picture shows a ball with built-in stopwatch that starts when the ball is dropped (release of the button) and stops on impact. It has onehundredth of a second precision. We have used this ball to indirectly measure the Planck length 100 times without knowledge of $G$ or $\hbar$ (as reported in Table 1.

the ball took to fall this distance and input this time into the formula 13. The formula also requires the reduced Compton wavelength of the Earth and this, as we have demonstrated in section II, can be found independent of any knowledge of $G$ or $h$. Table 1 reports the 100 ball-drop times and the corresponding Planck length we obtain from formula 13. Our average value from 100 ball-drop measurements is $1.56 \times 10^{-35} \mathrm{~m}$. Almost all these values are somewhat lower than the CODATA 2019 value for the Planck length, which is $1.616255 \times 10^{-35} \mathrm{~m}$. That our predictions from such measurements are lower is partly to be expected-first of all, the formula we use is only valid for an ideal ball drop when the ball is not rotating at all during the drop, and secondly that the drop is performed in a vacuum. Both these effects give a longer time and lower Planck length estimate than under ideal conditions. We have merely performed the ball drops in a library room at normal room temperature. Air drag will slow the ball-drop time somewhat. After adjusting for air drag [30] we get an average estimated Planck length of approximately $1.59 \times 10^{-35}$ meters. The theoretical time for the fall including air drag is given by the formula:

$$
t=\sqrt{\frac{m}{g k}} \operatorname{acosh}\left(e^{H k / m}\right)
$$

where, in our case, the $m$ is the $\mathrm{kg}$ mass of the clock ball. This we measured on a kitchen weight to be 121 grams; further, $H$ is the height of the drop, as before; 2 meters, and $k=\frac{1}{2} \rho C_{d} 4 \pi r^{2}$, where $r$ is the radius of the drop ball that we measured to be 5 centimeters and $C_{d}$ is the drag coefficient that is 0.47 for a ball, and $\rho$ is the air density where we used $1.225 \mathrm{~kg} / \mathrm{m}^{3}$.

So, to perform the adjustment, we calculate the theoretical time for the drop in a vacuum minus the theoretical time in the air and adjust our time numbers for this difference. This gives the adjusted Planck length. Our Planck length measure of $1.59 \times 10^{-35}$ after air-drag adjustment is naturally still less accurate than the value given by CODATA 2019, which is obtained from very accurate measurements with expensive apparatus that has been used to measure $G$, that again has been converted into the Planck length indirectly from dimensional analysis. Still, we found it interesting that we, with such very simple equipment, can measure the Planck length, which can easily be utilized in a classroom situation with minimal budget.

More importantly, we have demonstrated that this can be done without any knowledge of $G$. Our way to find the Planck length is also very easy to perform, based on the fact that we already know the Compton wavelength of the Earth. Naturally, an interesting question is why it is possible to measure the Planck length independent of $G$ and $\hbar$. One possibility is that $G$ is really a composite constant of the form $G=\frac{l_{p}^{2} c^{3}}{\hbar}$, as has been suggested by [31], [32], and that it is the Planck length and the speed of light (gravity) that are really important for gravity predictions and observations. Again, it is only after one solves the circular problem and is able to find the Planck length independent of $G$ that this approach can really be useful. The Planck constant, as we have seen, cancels out the Planck constant in the mass; something discussed in more detail in [37] when doing gravitational predictions. This would still mean $G$ is a universal gravitational constant, but that it consists of more fundamental constants, so $G$ is actually not needed. This is a new view and we naturally do not ask anyone to take this for granted, but rather to investigate it carefully. Recently we [37], [38] have argued that $G, h$ and $c$ can be replaced with only $c$ and $l_{p}$. Superstring theory [39] has also suggested that the speed of light $c$ and the Planck length are the two universal fundamental constants. However, superstring theory has not led to a way to find the Planck length independent of $G$, nor has it led to direct experimental evidence [40] of string theory. It is therefore in high time to look closer at other approaches, and we think that to find the Planck length independent of $G$ and $\hbar$ could be of great importance towards a unified quantum gravity theory.

\section{CONCLUSION}

The main contribution of this paper is that one can very easily measure the Planck length quite accurately with a simple and cheap device that is ideal for use in classroom settings. We hope this will encourage both researchers and students of physics to look closer at the Planck length, both from a theoretical and experimental standpoint.

We have demonstrated that the Planck length can be found independent of $G$ and $h$ simply by using a ball with a built-in stopwatch. This supports the idea that the Newton gravitational constant is likely a composite constant of the form $G=\frac{l_{p}^{2} c^{3}}{\hbar}$ where $\hbar$ is needed to cancel out the Planck constant in the $\mathrm{kg}$ definition of mass. Our more direct (but still indirect) way of finding the Planck length supports our theory that the detection of gravity is indeed the indirect detection of the Planck scale. We do not, however, assume this should or will be easily accepted. Still, such an idea should be of interest to the research community. After all, early on, it was even ridiculed that the Planck length had anything to do with gravity. Today, 
however, most researchers in the field of quantum gravity think the Planck scale is essential, but they have just not figured out exactly how as yet. We hope this paper can encourage more researchers to consider the implications of why we can find the Planck length without any reliance on $G$. We also hope this simple way of measuring the Planck length can encourage a future generation of physics students to become interested in the Planck scale.

\begin{tabular}{|c|c|c|c|c|c|c|c|c|}
\hline $\begin{array}{c}\text { Ball drop } \\
\text { observation }\end{array}$ & $\begin{array}{c}\text { Time } \\
\text { measured }\end{array}$ & $\begin{array}{l}\text { Planck length } \\
\text { estimate }\end{array}$ & $\begin{array}{c}\text { Ball drop } \\
\text { observation }\end{array}$ & $\begin{array}{c}\text { Time } \\
\text { measured }\end{array}$ & $\begin{array}{c}\text { Planck length } \\
\text { estimate }\end{array}$ & $\begin{array}{c}\text { Ball drop } \\
\text { observation }\end{array}$ & $\begin{array}{c}\text { Time } \\
\text { measured }\end{array}$ & $\begin{array}{c}\text { Planck length } \\
\text { estimate }\end{array}$ \\
\hline 1 & 0.65 & $1.59 \mathrm{E}-35$ & 34 & 0.66 & $1.56 \mathrm{E}-35$ & 67 & 0.67 & $1.54 \mathrm{E}-35$ \\
\hline 2 & 0.65 & $1.59 \mathrm{E}-35$ & 35 & 0.67 & $1.54 \mathrm{E}-35$ & 68 & 0.67 & $1.54 \mathrm{E}-35$ \\
\hline 3 & 0.67 & $1.54 \mathrm{E}-35$ & 36 & 0.67 & $1.54 \mathrm{E}-35$ & 69 & 0.66 & $1.56 \mathrm{E}-35$ \\
\hline 4 & 0.67 & $1.54 \mathrm{E}-35$ & 37 & 0.68 & $1.52 \mathrm{E}-35$ & 70 & 0.65 & $1.59 \mathrm{E}-35$ \\
\hline 5 & 0.64 & $1.61 \mathrm{E}-35$ & 38 & 0.66 & $1.56 \mathrm{E}-35$ & 71 & 0.67 & $1.54 \mathrm{E}-35$ \\
\hline 6 & 0.66 & $1.56 \mathrm{E}-35$ & 39 & 0.69 & $1.49 \mathrm{E}-35$ & 72 & 0.66 & $1.56 \mathrm{E}-35$ \\
\hline 7 & 0.66 & $1.56 \mathrm{E}-35$ & 40 & 0.67 & $1.54 \mathrm{E}-35$ & 73 & 0.67 & $1.54 \mathrm{E}-35$ \\
\hline 8 & 0.64 & $1.61 \mathrm{E}-35$ & 41 & 0.67 & $1.54 \mathrm{E}-35$ & 74 & 0.66 & $1.56 \mathrm{E}-35$ \\
\hline 9 & 0.65 & $1.59 \mathrm{E}-35$ & 42 & 0.67 & $1.54 \mathrm{E}-35$ & 75 & 0.68 & $1.52 \mathrm{E}-35$ \\
\hline 10 & 0.65 & $1.59 \mathrm{E}-35$ & 43 & 0.69 & $1.49 \mathrm{E}-35$ & 76 & 0.69 & $1.49 \mathrm{E}-35$ \\
\hline 11 & 0.64 & $1.61 \mathrm{E}-35$ & 44 & 0.64 & $1.61 \mathrm{E}-35$ & 77 & 0.65 & $1.59 \mathrm{E}-35$ \\
\hline 12 & 0.66 & $1.56 \mathrm{E}-35$ & 45 & 0.67 & $1.54 \mathrm{E}-35$ & 78 & 0.67 & $1.54 \mathrm{E}-35$ \\
\hline 13 & 0.67 & $1.54 \mathrm{E}-35$ & 46 & 0.65 & $1.59 \mathrm{E}-35$ & 79 & 0.66 & $1.56 \mathrm{E}-35$ \\
\hline 14 & 0.65 & $1.59 \mathrm{E}-35$ & 47 & 0.66 & $1.56 \mathrm{E}-35$ & 80 & 0.68 & $1.52 \mathrm{E}-35$ \\
\hline 15 & 0.66 & $1.56 \mathrm{E}-35$ & 48 & 0.67 & $1.54 \mathrm{E}-35$ & 81 & 0.67 & $1.54 \mathrm{E}-35$ \\
\hline 16 & 0.69 & $1.49 \mathrm{E}-35$ & 49 & 0.67 & $1.54 \mathrm{E}-35$ & 82 & 0.67 & $1.54 \mathrm{E}-35$ \\
\hline 17 & 0.67 & $1.54 \mathrm{E}-35$ & 50 & 0.64 & $1.61 \mathrm{E}-35$ & 83 & 0.68 & $1.52 \mathrm{E}-35$ \\
\hline 18 & 0.67 & $1.54 \mathrm{E}-35$ & 51 & 0.67 & $1.54 \mathrm{E}-35$ & 84 & 0.67 & $1.54 \mathrm{E}-35$ \\
\hline 19 & 0.67 & $1.54 \mathrm{E}-35$ & 52 & 0.69 & $1.49 \mathrm{E}-35$ & 85 & 0.69 & $1.49 \mathrm{E}-35$ \\
\hline 20 & 0.68 & $1.52 \mathrm{E}-35$ & 53 & 0.67 & $1.54 \mathrm{E}-35$ & 86 & 0.68 & $1.52 \mathrm{E}-35$ \\
\hline 21 & 0.66 & $1.56 \mathrm{E}-35$ & 54 & 0.65 & $1.59 \mathrm{E}-35$ & 87 & 0.63 & $1.64 \mathrm{E}-35$ \\
\hline 22 & 0.66 & $1.56 \mathrm{E}-35$ & 55 & 0.65 & $1.59 \mathrm{E}-35$ & 88 & 0.65 & $1.59 \mathrm{E}-35$ \\
\hline 23 & 0.65 & $1.59 \mathrm{E}-35$ & 56 & 0.66 & $1.56 \mathrm{E}-35$ & 89 & 0.64 & $1.61 \mathrm{E}-35$ \\
\hline 24 & 0.67 & $1.54 \mathrm{E}-35$ & 57 & 0.67 & $1.54 \mathrm{E}-35$ & 90 & 0.65 & $1.59 \mathrm{E}-35$ \\
\hline 25 & 0.66 & $1.56 \mathrm{E}-35$ & 58 & 0.68 & $1.52 \mathrm{E}-35$ & 91 & 0.66 & $1.56 \mathrm{E}-35$ \\
\hline 26 & 0.66 & $1.56 \mathrm{E}-35$ & 59 & 0.64 & $1.61 \mathrm{E}-35$ & 92 & 0.66 & $1.56 \mathrm{E}-35$ \\
\hline 27 & 0.67 & $1.54 \mathrm{E}-35$ & 60 & 0.67 & $1.54 \mathrm{E}-35$ & 93 & 0.65 & $1.59 \mathrm{E}-35$ \\
\hline 28 & 0.65 & $1.59 \mathrm{E}-35$ & 61 & 0.66 & $1.56 \mathrm{E}-35$ & 94 & 0.65 & $1.59 \mathrm{E}-35$ \\
\hline 29 & 0.69 & $1.49 \mathrm{E}-35$ & 62 & 0.67 & $1.54 \mathrm{E}-35$ & 95 & 0.66 & $1.56 \mathrm{E}-35$ \\
\hline 30 & 0.65 & $1.59 \mathrm{E}-35$ & 63 & 0.66 & $1.56 \mathrm{E}-35$ & 96 & 0.66 & $1.56 \mathrm{E}-35$ \\
\hline 31 & 0.67 & $1.54 \mathrm{E}-35$ & 64 & 0.67 & $1.54 \mathrm{E}-35$ & 97 & 0.65 & $1.59 \mathrm{E}-35$ \\
\hline 32 & 0.67 & $1.54 \mathrm{E}-35$ & 65 & 0.67 & $1.54 \mathrm{E}-35$ & 98 & 0.67 & $1.54 \mathrm{E}-35$ \\
\hline \multirow[t]{4}{*}{33} & 0.67 & $1.54 \mathrm{E}-35$ & 66 & 0.67 & $1.54 \mathrm{E}-35$ & 99 & 0.66 & $1.56 \mathrm{E}-35$ \\
\hline & & & & & & 100 & 0.65 & $1.59 \mathrm{E}-35$ \\
\hline & Average time : & 0.664 & & & & Average & Planck length : & $1.56 \mathrm{E}-35 \mathrm{~m}$ \\
\hline & Air-drag adjusted time: & 0.640 & & & & Air drag adjusted & Planck length : & 1.59E-35 m \\
\hline
\end{tabular}

TABLE I

ONE HUNDRED PLANCK LENGTH MEASUREMENTS FROM USING A STOP-CLOCK BALL. DROP HEIGHT TWO METERS, REDUCED COMPTON WAVELENGTH USED FOR THE EARTH $5.89 \times 10^{-68} \mathrm{~m}$. BE AWARE THAT THIS CAN BE FOUND WITHOUT KNOWLEDGE OF $G$ OR $\hbar$. 


\section{REFERENCES}

[1] M. Planck. Natuerliche Masseinheiten. Der Königlich Preussischen Akademie Der Wissenschaften, 1899.

[2] M. Planck. Vorlesungen über die Theorie der Wärmestrahlung. Leipzig: J.A. Barth, p. 163, see also the English translation "The Theory of Radiation" (1959) Dover, 1906

[3] J. G. Stoney. On the physical units of nature. The Scientific Proceedings of the Royal Dublin Society, 3, 1883.

[4] A. Einstein. Näherungsweise integration der feldgleichungen der gravitation. Sitzungsberichte der Königlich Preussischen Akademie der Wissenschaften Berlin, 1916.

[5] A. S. Eddington. Report On The Relativity Theory Of Gravitation. The Physical Society Of London, Fleetway Press, London, 1918.

[6] P. W. Bridgman. Dimensional Analysis. New Haven: Yale University Press, 1931.

[7] A. C. Mead. Possible Connection Between Gravitation and Fundamental Length Phys. Rev., 135(38), $1964 . \quad$ URL https://link.aps.org/doi/10.1103/PhysRev.135.B849.

[8] T. Padmanabhan. Planck length as the lower bound to all physical length scales. General Relativity and Gravitation, 17, 1985. URL https://doi.org/10.1007/BF00760244.

[9] S. Hossenfelder. Can we measure structures to a precision better than the Planck length? Classical and Quantum Gravity, 29, 2012. URL https://doi.org/10.1088/0264-9381/29/11/115011.

[10] S. Hossenfelder. Minimal length scale scenarios for quantum gravity. Living Reviews in Relativity, 16, 2013. URL https://doi.org/10.12942/lrr2013-2.

[11] G. M. Obermair. Primordial Planck mass black holes (PPMBHS) as candidates for dark matter? Journal of Physics, Conference Series, 442 , 2013. URL https://doi.org/10.1088/1742-6596/442/1/012066.

[12] V. Faraoni. Three new roads to the Planck scale American Journal of Physics, 85, $2017 . \quad$ URL https://aapt.scitation.org/doi/pdf/10.1119/1.4994804.

[13] A. Unzicker. The Mathematical Reality: Why Space and Time Are an Illusion. Independently published, 2020.

[14] E. G. Haug. Can the Planck length be found independent of big $G$ ? Applied Physics Research, 9(6):58, 2017. URL https://doi.org/10.5539/apr.v9n6p58.

[15] E. G. Haug. Finding the Planck length multiplied by the speed of light without any knowledge of $G, c$, or $h$, using a Newton force spring. Journal Physics Communication, 4:075001, 2020. URL https://doi.org/10.1088/2399-6528/ab9dd7.

[16] E. G. Haug. Using a grandfather pendulum clock to measure the world's shortest time interval, the Planck time (with zero knowledge of $G$ ). Journal of Applied Mathematics and Physics, 9:1076, 2021c.

[17] A. H. Compton. A quantum theory of the scattering of $x$ rays by light elements. Physical Review, 21(5):483, 1923. URL https://doi.org/10.1103/PhysRev.21.483.

[18] G. Gräff, H. Kalinowsky, and J. Traut. A direct determination of the proton electron mass ratio. Zeitschrift für Physik A Atoms and Nuclei, 297 (1), 1980. URL https://link.springer.com/article/10.1007/BF01414243.

[19] R.S. Van-Dyck, F.L. Moore, D.L. Farnham, and P.B. Schwinberg. New measurement of the proton-electron mass ratio. International Journal of Mass Spectrometry and Ion Processes, 66(3), 1985. URL https://doi.org/10.1016/0168-1176(85)80006-9.

[20] L.S. Levitt. The proton Compton wavelength as the 'quantum' of length. Experientia, 14:233, 1958. URL https://doi.org/10.1007/BF02159173.

[21] O. L. Trinhammer and H. G. Bohr. On proton charge radius definition. EPL, 128:21001, 2019. URL https://doi: 10.1209/0295-5075/128/21001.

[22] E. Massam and G. Mana. Counting atoms. Nature Physics, 12:522, 2016. URL https://doi.org/10.1038/nphys3754.

[23] P. Becker and H. Bettin. The Avogadro constant: determining the number of atoms in a single-crystal $28 \mathrm{Si}$ sphere. Phil. Trans. R. Soc. A, 369 3925, 2011. URL https://doi:10.1098/rsta.2011.0222.

[24] P. Becker. The new kilogram definition based on counting the atoms in a ${ }^{28} \mathrm{Si}$ crystal. Contemporary Physics, 53:461, 2012. URL https://doi.org/10.1080/00107514.2012.746054

[25] I Newton. Philosophiae Naturalis Principia Mathematica. London, 1686.

[26] H. Cavendish. Experiments to determine the density of the earth. Philosophical Transactions of the Royal Society of London, (part II), 88, 1798.

[27] Bartl, G. et. al. A new ${ }^{28} \mathrm{Si}$ single crystal: Counting the atoms for the new kilogram definition. Metrologica, 54:693, 2017. URL https://doi.org/10.1088/1681-7575/aa7820.
[28] A. Cornu and J. B. Baille. Détermination nouvelle de la constante de l'attraction et de la densité moyenne de la terre. C. R. Acad. Sci. Paris, $76,1873$.

[29] C. V. Boys. On the Newtonian constant of gravitation. Nature, 5, 1894. URL https://doi.org/10.1038/050330a0.

[30] R. Cross. Measuring the drag force on a falling ball. The Physics Teacher, 52(169), 2014. URL https://doi.org/10.1119/1.4865522.

[31] E. G. Haug. The gravitational constant and the Planck units. A simplification of the quantum realm. Physics Essays, 29(4):558, 2016. URL https://doi.org/10.4006/0836-1398-29.4.558.

[32] E. G. Haug. Collision space-time: Unified quantum gravity. Physics Essays, 33(1):46, 2020a. URL https://doi.org/10.4006/0836-1398-33.1.46.

[33] K. Cahill. Tetrads, broken symmetries, and the gravitational constant Zeitschrift Für Physik C Particles and Fields, 23:353, 1984.

[34] E. R. Cohen. Graviton Exchange and the Gravitational Constant in the book Gravitational Measurements, Metrology and Constants. Edited by Sabbata, V. and Gillies, G. T. and Melniko, V. N., Netherland, Kluwer Academic Publishers, 1987.

[35] M. E. McCulloch. Quantised inertia from relativity and the uncertainty principle. Europhysics Letters (EPL), 115(6):69001, 2016. URL https://doi.org/10.1209/0295-5075/115/69001.

[36] E. G. Haug. Newton and Einstein's gravity in a new perspective for Planck masses and smaller sized objects. International Journal of Astronomy and Astrophysics, 8, 2018. URL https://doi.org/10.4236/ijaa.2018.81002.

[37] E. G. Haug. Demonstration that Newtonian gravity moves at the speed of light and not instantaneously (infinite speed) as thought! Journal of Physics Communication., 5(2):1, 2021. URL https://doi.org/10.1088/2399-6528/abe4c8.

[38] E. G. Haug. Quantum Gravity Hidden in Newton Gravity And How To Unify It With Quantum Mechanics. in the book: The Origin of Gravity from the First Principles, Editor Volodymyr Krasnoholovets, NOVA Publishing, New York, 2021b.

[39] M. J. Duff, L. B. Okun, and G. Veneziano. Trialogue on the number of fundamental constants. Journal of High Energy Physics, 2002, 2002. URL https://doi.org/10.1088/1126-6708/2002/03/023.

[40] J. Conlon. Why String Theory? CRC Press, 2015. 